\title{
Unraveling deformation mechanisms around FCC and BCC nanocontacts through slip trace and pileup topography analyses
}

\author{
Javier Varillas ${ }^{1,3}$, Jan Očenášek ${ }^{1}$, Jordi Torner ${ }^{2}$, Jorge Alcalá ${ }^{3}$ \\ ${ }^{1}$ New Technologies Research Centre, University of West Bohemia in Pilsen, 30614 Plzeň, \\ Czech Republic. \\ ${ }^{2}$ Department of Engineering Design, InSup, EEBE. Universitat Politècnica de Catalunya, \\ 08019 Barcelona, Spain. \\ ${ }^{3}$ Department of Materials Science and Metallurgical Engineering, InSup, ETSEIB. \\ Universitat Politècnica de Catalunya, 08028 Barcelona, Spain.
}

\begin{abstract}
Nanocontact loadings offer the potential to investigate crystal plasticity from surface slip trace emissions and distinct pileup patterns where individual atomic terraces arrange into hillocks and symmetric rosettes. Our MD simulations in FCC Cu and Al nanocontacts show development of specific dislocation interception, cross-slip and twin annihilation mechanisms producing traces along characteristic $<011>$ and $<112>$ directions. Although planar slip is stabilized through subsurface dislocation interactions, highly serrated slip traces always predominate in Al due to the advent of cross-slip of the surfaced population of screw dislocations, leading to intricate hillock morphologies. We show that the distinct wavy hillocks and terraces in BCC Ta and Fe nanocontacts are due to dislocation kinking and outward spreading of surfaced screw segments, which originate from dislocation loops induced by twin annihilation and twin-mediated nucleation processes in the subsurface. While increasing temperature favors terrace formation in BCCs, surface decorations are enhanced in FCCs limiting hillock definition. It is found that material bulging against the indenter-tip is a distinctive feature in nanocontact plasticity associated with intermittent defect bursts. Bulging is enhanced by recurrent slip traces introduced throughout the contact surface, as in the case of the strongly linear defect networks in FCC Al, and by specific twin arrangements at the vicinity of BCC nanocontacts. Defect patterning also produces surface depressions in the form of vertexes around FCC nanoimprints. While the rosette morphologies are consistent with those assessed experimentally in greater FCC and BCC imprints, topographical pileup due to extensive bulging becomes prominent at the nanoscale.
\end{abstract}

Keywords: Nanocontacts, slip traces, indentation pileup, indentation rosettes, nanotwins

Submitted to Acta Materialia

August 2016 


\section{Introduction}

Surface slip trace emission is a fundamental manifestation from crystal plasticity. Slip trace emergence is concomitant to indentation testing, where plastic patterning leads to material pileup in the form of atomic terraces clustering into hillocks and symmetric rosette arrangements. Onset of different hillock topographies has been systematically reported in face-centered cubic (FCC) and body-centered cubic (BCC) metallic surfaces (e.g., [1-9]) as well as in ceramic surfaces (e.g., [10-12]).

Understanding of plastic patterning is imposing because of its essential multiscale character, where dislocation slip and twinning come into play to produce entangled defect networks underneath the imprint [13] from which the pileup topographies originate. Patterning is intrinsically size-dependent, involving preexisting networks in large imprints or incepted networks under extreme pressures in minute nanoimprints. Onset of size-dependent deformation mechanisms, as twinning in FCC nanocrystals ([14,15]), may tentatively influence nanoscale patterning. Following onset of planar vs. wavy hillock morphologies and the development of different rosette symmetries in FCCs and BCCs $[5,6,8]$, patterning arises as a crystalline-dependent phenomenon that is potentially affected by temperature, applied contact pressure and specific defect annihilation mechanisms at the surface $[16,17]$.

The objective of this work is to investigate slip trace patterning through comprehensive Molecular Dynamics (MD) simulations of nanocontact plasticity across a wide temperature range in different surface orientations. It is our purpose to furnish a fundamental understanding into the role of FCC vs. BCC plasticity mechanisms upon the development of different hillock morphologies and rosette symmetries. In particular, we seek to study how patterning is affected by (i) competition between twinning and dislocation slip, (ii) the stacking-fault energy that rules dislocation cross-slip in FCCs, and (iii) dislocation kinking in BCCs. 
While the majority of investigations on nanocontact-induced plasticity concern analyses of the applied load-penetration depth $\left(P-h_{\mathrm{s}}\right)$ curves in connection with defect network inceptions, little is known about defect patterning processes leading to material pileup (plastic bulging) at the nanocontact periphery. Since plastic bulging is a fingerprint of the mechanical response fundamentally contributing to imprint formation [18-20], it is our objective to investigate the associated micromechanisms in FCC and BCC surfaces. We also seek to quantify plastic bulging in nanoimprints and to compare the results against those from greater imprint sizes.

\section{Computational methods}

The MD simulations were carried-out with the LAMMPS code under the Canonical (NVT) statistical ensemble using the Nosé-Hoover thermostat with timestep ranging from 1 to 3 fs. The indented crystals were modelled through the embedded-atom method (EAM) potentials by Mishin et al. for $\mathrm{Cu}$ and $\mathrm{Al}$ [21], and the EAM potentials constructed by Li et al. for Ta [22] and Mendelev et al. for Fe [23]. Such EAM potentials have been extensively used in previous investigations of nanocontact plasticity [24-31].

The indenter was modelled with a repulsive potential reproducing the displacement field of a spherical tip against a flat surface. The force applied to each surface atom is [32]

$$
F=-K(D / 2-\delta)^{2}
$$

where $K$ is the indenter stiffness, $D$ is the indenter diameter and $\delta$ is the distance from the atom to the center of the repulsive sphere. The indenters had $D=48$ and $24 \mathrm{~nm}$ and were brought into contact to a maximum ratio between contact radius and tip diameter, $a / D$, of 0.35 at a loading rate of $4 \mathrm{~m} / \mathrm{s}$. Stiffness $K$ was set at $100 \mathrm{eV} / \AA^{3}$ where the resulting total applied indentation load $(P)$ - penetration depth $\left(h_{\mathrm{s}}\right)$ curves for the early elastic contact responses are in accordance with those from complementary coarse-grained anisotropic linear-elastic finite element analyses (FEA). [The physically realistic range of 
$K$ values is wide, as similar contact responses attain within $K=1-100 \mathrm{eV} / \AA^{3}[33,34]$. Increasing computational fluctuations in the $P-h_{\mathrm{s}}$ curves arise for $K>500 \mathrm{eV} / \AA^{3}$.] The indented domains had cuboidal shapes with top (indented) surfaces oriented along the [001], [011] and [111] directions. Periodic boundaries were applied to the four lateral sides while the atomic positions remain fixed for the bottom side. The MD domains indented with $D=48 \mathrm{~nm}$ were of 70 (length) $\times 70$ (width) $\times 40$ (height) $\mathrm{nm}^{3}$; containing $\approx 10 \times 10^{6}$ atoms for Ta and $\mathrm{Fe}, \approx 11 \times 10^{6}$ atoms for $\mathrm{Al}$, and $\approx 16 \times 10^{6}$ atoms for $\mathrm{Cu}$. One massive simulation with $D=100 \mathrm{~nm}$ was performed for a $(001) \mathrm{Cu}$ surface at $77 \mathrm{~K}$ (MD box of $105 \times 105 \times 40 \mathrm{~nm}^{3}$ with $\approx 40 \times 10^{6}$ atoms). Further simulations for $D=24$ $\mathrm{nm}$ comprised domains of $36 \times 36 \times 16 \mathrm{~nm}^{3}$; containing $\approx 1.3 \times 10^{6}$ atoms for Ta and $\approx$ $1.8 \times 10^{6}$ atoms for $\mathrm{Cu}$. All MD boxes were built at $\approx 0 \mathrm{~K}$ and allowed to minimize energy by increasing temperature towards the target indentation value at a rate of $1 \mathrm{~K} / \mathrm{ps}$ with timestep of $0.5 \mathrm{fs}$.

The indentation response was modelled at $77 \mathrm{~K}$ for the differently oriented $\mathrm{Cu}$, $\mathrm{Ta}$ and $\mathrm{Fe}$ surfaces. Further simulations were conducted at $400 \mathrm{~K}$ for $\mathrm{Cu}$ and at $900 \mathrm{~K}$ for Ta surfaces, yielding the same homologous temperature of $0.3 \times T_{\mathrm{m}}$ for both metals (where $T_{\mathrm{m}}$ is the melting temperature). The role of cross-slip upon defect patterning was investigated by comparing the results from moderate stacking-fault energy $\mathrm{Cu}$ against a complete set of simulations performed in high stacking-fault energy Al at $300 \mathrm{~K}$.

Delaunay triangulation was used to compute the surface-projected contact area $A$ from the atomic coordinates, where the atoms in contact fulfill $F>0$ and $\delta \leq D / 2$ in Eq. (1). The associated contact radius and tip penetration were then obtained from $a=\sqrt{A / \pi}$ and $h_{\mathrm{c}}=(D / 2)-\sqrt{\left(D^{2} / 4\right)-a^{2}}$, respectively. Note that penetration depth measured from the free surface $h_{\mathrm{s}}$ differs from the above penetration depth $h_{\mathrm{c}}$ (Section 4.1 and Fig. 7(a)). 


\section{Mechanisms for the onset of slip traces}

\subsection{FCC nanocontacts}

Slip trace formation involves sweeping across the surface of dislocation segments decomposed into leading and trailing Shockley partials. A usual configuration is shown in Fig. 1(a) for the gliding of a surfaced screw segment with dislocation line vector $\xi$. The Burgers vector $b$ is the sum of the individual Burgers vectors from the constituting partials $\left(b_{1}+b_{2}\right)$, where $\xi \| b$ so as to produce a trace of varying height depending on the angle between $b$ and the surface normal. The equivalent pure edge construction, $\xi \perp$ $b$, concerns surfacing of the same dislocation line $\xi=\xi_{1}=\xi_{2}$ as in Fig. 1 (a), where net vector $b$ is now rather parallel to the surface so that the step produced by the leading partial tends to vanish as the trailing partial sweeps behind. Our simulations show that while such edge dislocation lines emerge at the surface, the largest dislocation population has the predominantly screw character producing large trace heights.

Preferential slip traces along $<112>$ and $<011>$ directions, marked in yellow in Fig. 2, naturally arise as a consequence of dislocation gliding in $\{111\}$ planes intercepting the surface (Fig. 1(a)). In (011) surfaces (Fig. 2(b)), dislocation gliding involves slip planes that form $35.3^{\circ}$ and $90^{\circ}$ with the indented surface, producing $<011>$ and $<112>$ traces, respectively. In (001) surfaces (Fig. 2(a)), the traces are directed along $<011>$ directions forming $54.7^{\circ}$ with the surface. This is because the indented $\{001\}$ planes cannot possibly contain $<112>$ traces. The $<112>$ slip traces are also missing in (111) surfaces (Fig. 2(c)) that may only contain $<011>$ traces forming $70.5^{\circ}$ with the $\{111\}$ slip planes.

Incipient dislocation surfacing occurs during the outward glide of prismatic loops nucleated underneath the indenter (Fig 1(b)). With increasing penetration, dislocation emissions from the entangled defect network in the subsurface contributes to further slip trace formation (Fig. 1(f)). A distinctive dislocation structure developing in all surface 
orientations involves two or more segments, joined in the subsurface through stable collinear interactions, whose outward glide produces parallel slip traces (Fig. 1(h)).

Nanoscopic twins are also found to induce marked slip traces in low stacking-fault energy $\mathrm{Cu}$ (Figs. 1(c), 1(h) and 2). Twin nucleation in FCCs is triggered under the large strain gradients and stresses imposed by the nanoindenter tip $([15,35,36])$. This involves successive emission of leading partial dislocations at the vicinity of the indenter so as to form the distinctive arrangement of parallel $\{111\}$ twined planes. Our simulations show that the twin boundaries are normal to the surface for (011) indentation, and inclined at $54.7^{\circ}$ and $70.5^{\circ}$ with respect to the surface for (001) and (111) indentations, respectively (Fig. 1(c)). The twinning crystallography ensures that all induced $<011>$ and $<112>$ traces simultaneously lie at the indented plane and a specific $\{111\}$ habit plane.

Twin annihilation entails when the energy associated with large stacking-fault ribbons favors nucleation of mating trailing partials in subsequent $\{111\}$ planes (Figs. 1(d) and 1(e)). Twin removal (i.e., detwinning) is produced by gliding of trailing partials at the twin boundaries, gradually introducing individual dislocation lines at the surface (Fig. 1(e)). Annihilation of large monatomic twins is commonly encountered.

The remarkably planar-like character of the defect network in $\mathrm{Cu}$ as compared to that in Al becomes evident from Figs. 3(f) and 3(g), where nanotwins are seldom formed in the latter because of its large stacking-fault energy.

\subsection{The role of dislocation cross-slip}

Serrated slip in FCCs involves cross-slip of surfaced dislocation lines. This requires constriction of partnering leading and trailing partials, producing a full non-dissociated screw segment that can freely glide in the intercepting cross-slip planes (Fig. 3(a) and 3(b)). Although cross-slip naturally furnishes serrated traces, local cross-slip events may introduce seemingly straight traces along the non-crystallographic $<001>$ slip directions 
marked in red in Fig. 2. Associated mechanisms are illustrated in Figs. 3(c)-(e)). [Note that such straight traces could not possibly develop in the absence of cross-slip since following Section 3.1, none of the $<001>$ traces can simultaneously lie on a $\{111\}$ slip plane and the indented $\{001\},\{011\}$ or $\{111\}$ surfaces.]

Onset of serrated traces is strongly favored in $\mathrm{Al}$ as compared to in $\mathrm{Cu}$ because the much greater stacking-fault energy of the former facilitates dislocation constrictions. While cross-slip is therefore general to FCCs, it becomes more evident in Al where the totality of the surfaced screw dislocation population exhibits serrated glide. Increasing temperature from $77 \mathrm{~K}$ to $400 \mathrm{~K}$ in $\mathrm{Cu}$ surfaces does not significantly change the length of the stacking fault ribbons so that the planar nature of the traces is maintained. Such a temperature raise, however, produces increasing surface decorations (Section 4.3).

A mechanism that hinders cross-slip in FCCs is the straightening of surfaced dislocation segments either by the pinning action exerted by the dislocation forest in the subsurface (Figs. 3(c)-(e)) or by the collinear interactions marked "iii" in Fig. 1(h).

The hillock morphologies emerging in FCCs through the above surface defect glide mechanisms are shown in Fig. 4 (see Section 4.3 for a detailed discussion).

\subsection{BCC nanocontacts}

Low-temperature simulations for $\mathrm{BCC}$ Ta and Fe show that twinning predominates in the highly deformed (core) region underneath the indenter-tip. Dislocation loops however develop with increasing tip penetration at the indentation core as well as throughout its vicinity (Fig. 5(a)). This feature varies depending on surface orientation, where in the case of (111) surfaces, the nucleated three-fold twin structure always prevails irrespective of penetration (Figs. 5(h) and 5(i)). A mechanism for twin-mediated emissions of dislocation loops involves propagation of partial (detwinning) dislocations across the $\{112\}$ boundaries of the precursory twins. The released (surface truncated) dislocation 
loops are comprised by two screw segments emitted sideways from the precursory twin, and one frontal predominantly edge dislocation produced along the $<111>$ growth direction of the twin (Fig. 5(a)) [17]. The reduced mobility of the laterally emitted screws hinders loop expansion while the frontal edge segment rapidly glides forward [37]. As opposed to the conventional kink-pair mechanism, the motion of the screws usually involves nucleation of a leading kink at the surface, which then spreads along the screw line. This is followed by surface nucleation and spreading of a second kink that trails behind, producing advancement of the screw segment by one Peierls barrier [38]. Wavy traces are introduced by the zigzag motion of the screws at the surface.

Another important mechanism for slip trace emission involves heterogeneous defect nucleation at the surfaces of precursory twins (Fig. 5(b)). Although direct dislocation loop nucleation from the precursory twins is usually detected, emission of secondary twins may also entail. [Such secondary twins rapidly annihilate thus emitting further dislocations loops.] When the Burgers vector from the edge segments of the loops has net upward component, surface interceptions induces planar traces (Fig. 5(c)) which are then extended in a wavy fashion through kinking of the partnering screws (Fig. 5(d)).

At elevated temperatures, homogeneous dislocation loop nucleation is promoted (without twin mediation), incepting highly linear dislocation networks at the indentation core (Fig. 5(e)). Expansion of the surface truncated loops is again limited by the reduced mobility of the screw segments, where the conventional kink-pair nucleation mechanism [38] becomes more prominent and stochastically occurs along the lines. The screw lines acquire a wavier appearance as compared to that at smaller temperatures, a feature that appears to favor the kink-pair nucleation mechanism. Since the screw segments intercepting the surface from a given dislocation loop have the same Burgers vector $b$ and opposite dislocation line vector $\xi$, they become mutually attracted and finally 
annihilate (Figs. 5(f) and 5(g)). This facilitates development of closed wavy terraces further injecting dislocation loops in the subsurface [30].

At increasing penetrations and elevated temperatures, surface truncated dislocation loops are also introduced through unzipping of dislocation lines from the dense linear defect network in the subsurface. These processes produce large amounts of traces with planar or wavy morphologies depending on whether the surface emitted segments have edge or screw characters, respectively.

Characteristic pileup topographies for BCCs are shown in Fig. 6 (see Section 4.3).

\section{Defect localization and patterning around nanoimprints}

\subsection{Deformation state at the imprint vicinity}

Bulging against the indenter tip is conventionally assessed through parameter $c^{2}$, defined as the ratio between the two penetration depths marked in Fig. 7(a) [18-20]:

$$
c^{2}=h_{\mathrm{c}} / h_{\mathrm{s}} \text {. }
$$

Parameter $c^{2}$ thus measures the vertical location of the contact boundary (along the $z$ axis) with respect to the total imposed indenter-tip penetration. Following Fig. 7, $c^{2}$ can be computed for (i) each individual material point around the contact boundary (i.e., throughout the circumferential $\phi$-axis), yielding parameter $c_{\phi}^{2}$; (ii) the vertical location of the effective (mean) contact radius $a$ (Section 2), yielding parameter $\overline{c^{2}}$; and (iii) the location of the maximum pileup height at the vicinity of the contact boundary upon tip removal, where $c_{\text {max }}^{2} \equiv h_{\mathrm{c}, \max } / h_{\mathrm{s}}$. While the above mean and local values of $c^{2}$ are under the applied indentation load, presently defined parameter $c_{\max }^{2}$ accounts for the elasto-plastic rebound of the surface produced upon the unloading indentation stage.

Extreme sinking-in attains within the elastic regime, where the mean value of $\overline{c^{2}}=0.5$ as contact entails only throughout the bottom $50 \%$ of the imposed tip penetration [18]. This is consistent with present simulations for all elastically deformed FCC and BCC 
surfaces (Fig. 8). A sustained raise of $\overline{c^{2}}$ from 0.5 indicates onset of plastic bulging with increasing tip penetration, marking interplay between elasticity and nanoscale plasticity (Fig. 8). A cross-sectional view of the displacement field is shown in Fig. 9, illustrating that all atoms displace downward during elasticity while the trajectory of the surface atoms changes to one with net upward component (uplift) during plastic deformation.

Figure $8(a)$ demonstrates that the evolution of $\overline{c^{2}}$ with penetration in the nanoscale is a highly discontinuous process associated with the onset of plastic bursts (pop-in events) in the applied load $(P)$-penetration depth $\left(h_{\mathrm{s}}\right)$ curves. Sudden increases in material bulging (encasing the indenter-tip) are thus attendant with a surging of slip traces. Surface orientations exhibiting pronounced first pop-ins also undergo dense defect network development as measured through a marked raise in $\overline{c^{2}}$ from the elastic value of $\approx 0.5$. For such orientations, the incipient elastic loading stage also spans over greater penetrations, which increases the elastic energy stored in the material and the abruptness of subsequent defect inception. Intermittent raises in $\overline{c^{2}}$ are further displayed throughout the entire penetration process, where any given abrupt raise in this parameter is directly linked with the occurrence of individual pop-ins in the $P-h_{\mathrm{s}}$ curves; the larger the load drop, the greater the raise in $c^{2}$ (marked points in Fig. 8(a)). The evolution of $\overline{c^{2}}$ with penetration is therefore governed by the pop-in distribution, which varies as a function of surface orientation, crystalline structure and temperature.

The large difference between the mean value along which $c_{\phi}^{2}$ fluctuates and the levels of $c_{\max }^{2}$ found for the different surfaces becomes evident in Figs. $7(\mathrm{~b})$ and $7(\mathrm{c})$, further illustrating that extreme pileup effects $\left(c_{\max }^{2}=1.3-1.5\right)$ are induced upon tip unloading.

\subsection{Defect mechanisms for plastic bulging}

Nanoscale plastic bulging in FCCs is greatly influenced by the onset of vertexes around the nanoimprints (Fig. 4), bounding topographical depressions from the surrounding 
uplifted surface. Vertex development emerges from twin localizations in low stackingfault energy $\mathrm{Cu}$, whereas the vertexes primarily involve recurrent dislocation slip in high stacking-fault energy Al. Although the FCC vertexes produce local minima of $c_{\phi}^{2}$ in Fig. 7(c), plastic localization in BCCs induces bulging and local peaks of $c_{\phi}^{2}$ in Fig. 7(b).

The following discussion concerns the role of the defect network underneath the nanoimprint upon the development of plastic bulging. First, notice that the level of $\overline{c^{2}}$ at maximum penetration $\left(h_{\mathrm{s}} / D \approx 0.12\right)$ for $(001)$ and $(011)$ Ta surfaces, Fig. $8(\mathrm{a})$, is smaller than that found in Al counterparts, Fig. 8(c). This suggests that the increase in defect mobility associated with the less entangled linear indentation core networks prevailing in high stacking-fault energy FCCs, enhances slip trace recurrence throughout the imprint as compared to in BCCs containing more stationary planar networks. [Note that $\overline{c^{2}}$ for $\mathrm{Cu}(011)$ and (001) surfaces exhibits an apparent plateau for $h_{\mathrm{s}} / D \approx 0.10-0.12$ (Fig. 8(b)). It thus follows that FCC crystals with low stacking-fault energy may not exhibit large values of $\overline{c^{2}}$ at deep penetrations (see Section 4.4).]

Increasing temperature in $\mathrm{BCC} \mathrm{Ta}$ and $\mathrm{Fe}$ facilitates development of highly mobile (linear) defect networks (Fig. 5(e)), shifting $\overline{c^{2}}\left(h_{\mathrm{s}} / D \approx 0.12\right)$ towards greater values in agreement with the above discussion. This is illustrated in Fig. 8(d) for Ta (011), where strong twin annihilation occurs at $900 \mathrm{~K}$. Since a temperature raise from 77 to $400 \mathrm{~K}$ in $\mathrm{Cu}$-resulting in the same homologous temperature as in Ta at $900 \mathrm{~K}$ - is not associated with significant variations in the balance between the planar/linear character of the network, the evolution of $\overline{c^{2}}$ remains essentially unaffected (Fig. 8(d)).

Our MD simulations for Ta (111) nanoimprints interestingly show that bulging may not be necessarily restrained by the onset of planar indentation core networks. For this surface orientation, large plastic slips attain due to the development of a three-fold twin structure 
(Fig. 5(h)), which strongly favors material bulging at surface projections from the preferential $<111>$ growth directions of the twins (Fig. 6(g)). Since the slip carried by the twins thus produces large surface steps, this counteracts for the overall lack of network mobility so that the evolution of $\overline{c^{2}}$ with penetration becomes similar to that measured in FCC Al. (Compare Figs. 8(a) and 8(c) past early defect inception -i.e., for $h_{s} / D>0.08$.)

\subsection{Mechanisms for hillock development}

Plastic bulging around the indenter tip and hillock development are two distinct manifestations of nanocontact plasticity. The former arises from the upward displacement field associated with defect network inception underneath the imprint (Section 4.1) in conjunction with slip trace emergence at the contact surface, $r \leq a$ (Section 4.2), whereas the latter is essentially due to patterning processes that introduce surface pileup some distance away from the contact boundary, $r>a$. This is evidenced in BCC surfaces where the hillocks faint for the (011) orientation even though parameter $\overline{c^{2}}$ increases from $\approx 0.50$ to 0.85 with tip penetration (see Figs. $6(\mathrm{c})$ and $8(\mathrm{a})$ ). While there is always good correlation between the locations of the hillocks and those for which maximum bulging (pileup) entails in FCCs, this is not necessarily fulfilled in BCCs as explained below.

Directing attention to Figs. 2 and 4, hillock formation in FCCs involves specific slip trace arrangements producing topographical pileup through (i) outwards gliding of dislocations from the imprint along $<112>$ and $<011>$ directions; (ii) cross-slip of such dislocations towards tangential $<112>$ and $<011>$ directions, which leads to serrated terrace patterns; and (iii) interceptions among surfaced dislocations producing terrace edges. Increasing temperature favors network intricacy and the emergence of a greater number of traces that produce decorated surfaces rather than arranging into specific pileup topographies. Consequently, the hillock patters in FCC Cu appear to be less defined (compare Figs. 4(b) and $4(\mathrm{c})$ ). The role of cross-slip upon the hillock morphology becomes evident by 
comparing Figs. 4(b) and 4(c) for Cu with Fig. 4(f) for high stacking-fault energy Al, where the enhancement of cross-slip in the latter yields highly serrated hillocks.

The plastic hillocks in BCC Ta and Fe usually involve wavy terrace formations because of the multiple slip systems where the predominantly screw surfaced dislocation population glide through kinking mechanisms. Ta (001) and (011) surfaces are characterized by mild terrace development, where the surface truncated loops are primarily produced through twin annihilation and heterogeneous nucleation processes, respectively (Section 3.3). For these orientations, the terraces are located at the surface projections of the preferential $<111>$ gliding direction of the dislocation loops in the subsurface, which coincides with the locations of maximum bulging. On the other hand, for the Ta (111) orientation, the surface truncated loops primarily arise through heterogeneous (twin-mediated) dislocation nucleation (Section 3.3). This orientation further exhibits remarkable lateral expansion of the surfaced screw segments, so that welldeveloped rosette arms form at the surface at $\sim 60^{\circ}$ from the locations where the bulged (twinned) regions emerged (see Figs. 6(f) and 6(g)).

At low temperatures, limited surface emergence and outward spreading of dislocations in BCCs is evidenced by the proximity of the hillocks to the contact boundary in (001) surfaces (Fig. 6(a)). Moreover, in the case of Ta (011), terrace formation vanishes at low temperatures as the heterogeneously nucleated dislocation loops remain in the subsurface (Fig. 6(c)). Increasing temperature favors attainment of highly linear defect networks emerging from recurrent homogeneous loop nucleation events (Fig. 5(e)) that facilitate terrace formation (Fig. 6(d)).

\subsection{Size effects and comparisons with experiments}

The presently found planar slip features and preferential $<011>$ and $<112>$ slip trace directions in FCC Cu reproduce experimental findings in low and moderate stacking fault 
energy crystals $[2,3,9]$. Moreover, the rosette arrangement in FCCs (Fig. 4) replicates the four-fold rotational symmetry from micro and nano-indentation experiments in (011) surfaces [4,39]. While the experimental results also show that a similar four-fold symmetry emerges in (001) FCC surfaces, this feature only becomes apparent through massive MD simulations performed with $D=100 \mathrm{~nm}$ (Fig. 4(h)). The simulations are not conclusive when it comes to mimic the experimentally found six-fold rotational symmetry of the rosettes in (111) surfaces [9], suggesting that such more elaborate patterns require enhanced defect clustering that may only attain for $D>100 \mathrm{~nm}$.

Our simulations reproduce the salient features of the hillock arrangements found from nanoindentation experiments in BCC Ta [6]. In the case of (001) and (011) surfaces, the four-fold rotational symmetries of the terraces along $<011>$ and $<111>$ directions in Figs. 6(a) and 6(d), respectively, closely match the experimentally observed morphologies. The elongated shape of the terraces (along the $\phi$-axis around the imprint) developing at the onset of defect inception from such nanoindentation experiments, is also in excellent agreement with that produced in the simulations (Fig. 6). Limited terrace formation is however characteristic of present BCC (001) and (011) nanocontacts as compared to the much more prominent rosettes found in greater BCC imprints [6].

The experimentally found rosette morphology in (111) Ta surfaces exhibits the characteristic three-fold rotational symmetry that is herein shown to arise because of the lateral expansion of the surface truncated loops (see Fig. 6(f) upon unloading and Section 4.3). Our results for such (111) surfaces are illustrative in that while pronounced defect patterning into rosette configurations may arise in $\mathrm{BCC}$ nanocontacts, the rosettes may lack some of the anticipated symmetrical features from greater imprints. While the location of any given two neighboring rosette arms from the experiments is consistent with that attaining in the MD simulations (see hillocks h1 and h3 in Fig. 6(g)), onset of 
the third arm cannot be replicated within the size of present nanoimprints. Statistical variations in the nucleation process is found to alter slip trace localization at the onset of defect inception, leading to the development of the third previously missing rosette arm at the expense of one of the other two arms. In light of the above discussion, the enhancement of slip trace recurrence and patterning produced with greater tips $(D>100$ nm) would tentatively produce the full three-arm rosette configuration.

The simulations also show that the early accumulation of elastic strains leads to marked surface rebound induced upon nanotip removal from maximum imposed penetration. Parameter $c_{\text {max }}^{2}$ thus becomes $\approx 1.4$ for $\mathrm{Cu}$ which is much greater than the level of $\approx 1$ found in greater (macroscopic) imprints in the unloaded state [19]. Enhancement of defect patterning upon nanotip removal is further illustrated by the development of hillock h2 in Fig. 6(f) (see Supplementary Material).

The good correspondence between surface topographies from MD simulations and experiments across the indentation scales suggests that irrespectively of the extreme hardness values and defect densities characterizing nanocontact plasticity, slip trace patterning is fundamentally governed by the same (size-independent) dislocation mechanisms -such as cross-slip and kinking of surfaced screw segments, and preferential slip system interactions. On the other hand, the presently found twin nucleation and annihilation mechanisms at the contact boundary -accurately reproducing distinctive bulging features from nanoindentation tests in (111) Ta [6]- are taken to exclusively represent nanocontact plasticity under extreme applied pressures.

Although a transition in the defect nucleation mechanism from slip to twinning may also arise depending on loading rate in FCCs [40], this may not significantly affect the evolutionary character of the defect network governing hillock development away from the contact boundary. Moreover, since the dislocation free-flight velocity is already 
several orders of magnitude greater than the displacement velocity of the tip, a hypothetical reduction to realistic (small) loading rates may not drastically change presently found defect glide mechanisms and slip trace patterning into hillock morphologies. Loading rate effects could thus be tentatively assimilated to those produced by a temperature increase in present simulations. In passing, note that the comparison between experiments and MD simulations in FCCs does not shed light into the debate on the transition between defect nucleation mechanisms, as both twinning and dislocation slip produce qualitatively similar vertex patterns (compare $\mathrm{Al}$ vs. $\mathrm{Cu}$ in Fig. 4 where twinning occurs in the latter while dislocation slip prevails in the former).

Finally, an interesting result is that $\overline{c^{2}}$ reaches similar values as those measured for greater imprints in FCC Cu at similar normalized penetrations $h_{s} / D[19,20]$, already indicating that extensive plastic flow is promoted at the nanocontact boundary (see Section 4.2). The apparent plateau in $\overline{c^{2}}$ (see Fig. $8(\mathrm{~b})$ for $\mathrm{Cu}$ at $h_{S} / D \approx 0.12$ ) is in agreement with continuum plasticity analyses performed for greater (macroscopic) indentations, where plastic bulging becomes a signature of the uniaxial strain hardening response: the greater the strain hardening, the smaller the saturation level for $\overline{c^{2}}$ (see Fig. 14 in Ref. [20]). Bulging saturation in $\mathrm{Cu}$ nanocontacts would be therefore indicative of the prominent strain hardening behavior of low stacking-fault energy FCC metals; yielding to a plateau in $\overline{c^{2}}$ at smaller penetrations than for BCC Ta and FCC Al distinguished by milder uniaxial strain hardening responses.

\section{Concluding remarks}

1. Nanocontact plasticity in FCC crystals leads to surface emissions of dislocations and nanotwins whose preferential glide along $<011>$ and $<112>$ directions produces slip traces. The surfaced dislocation segments have predominantly screw character and originate from (i) prismatic loops emitted during the incipient defect nucleation stage; (ii) 
junction unzipping processes occurring within the defect network; and (iii) recurrent nanotwin annihilation at the vicinity of the contact boundary. Increasing stacking-fault energy from that of pure $\mathrm{Cu}$ to $\mathrm{Al}$ facilitates cross-slip of the surfaced dislocations which enhances slip trace serrations. Cross-slip is affected by the straightening of the surfaced dislocation lines by the pinning action from the defect network in the subsurface. Depending on dislocation pinning, traces with globally straight appearance thus emerge along non-crystallographic $<001>$ directions. These are produced by short-range crossslip processes along the above $<011>$ and $<112>$ directions.

2. The slip traces in BCCs are the manifestation of the interplay between twinning and dislocation slip in the subsurface. At low temperatures, limited annihilation of the twin structure leads to the emergence of surface truncated dislocation loops lying in characteristic $\{112\}$ planes. Loop expansion proceeds so that the edge segments glide towards the $<111>$ frontal direction of the precursory twins and the screw segments expand sideways, usually intercepting the surface. Heterogeneous dislocation loop nucleation processes from the twin surfaces may also contribute strongly to the onset of slip traces. At increasing temperatures, homogeneous dislocation loop nucleation is favored (without twin mediation), which injects linear defect networks of greater mobility in the subsurface. Depending on temperature, two dislocation kinking mechanisms of the screw segments are found to produce characteristic wavy traces in BCCs.

3. Topographical pileup around FCC nanoimprints involves terrace patterning into hillock morphologies whose rather planar sides denote interception of $<011>$ and $<112>$ slip traces. Development of increasingly serrated terrace morphologies is governed by crossslip and recurrent dislocation interceptions. Hillock morphology is thus affected by the stacking-fault energy of the crystal, so that more complex hillock shapes attain in high stacking-fault energy $\mathrm{Al}$ as compared to in moderate stacking-fault energy $\mathrm{Cu}$. 
Dislocation organization into hillocks becomes less evident at elevated temperatures, where the highly entangled defect networks in the subsurface produce prominent surface decorations. While similar four-fold symmetric rosette patterns as in micrometer-sized imprints emerge in both FCC and BCC nanocontacts depending on surface orientation, this feature is affected by indenter diameter. In some orientations, complex slip trace patterning only attains with tip diameters $D \geq 100 \mathrm{~nm}$ at large normalized penetrations $\left(h_{s} / D\right)$ that are 1.5 times greater than those marking early defect inception.

4. Hillock waviness in BCCs is governed by dislocation kinking of the surfaced screw segments originating from the aforementioned dislocation loop emissions. Shift from homogeneous to heterogeneous dislocation loop nucleation is ruled by surface orientation and temperature. Terrace formation is mild in (001) and (011) indentations, emerging along the preferential $<111>$ expanding direction of the defects underneath the imprint. Well-developed hillocks however arise away from the contact boundary in (111) indentations, where the surfaced screw dislocation population pronouncedly spreads outward from the imprint. Increasing temperature in BCCs favors onset of linear defect networks that always enhance terrace formation. Finally, it is found that characteristic extreme material uplift induced during nanotip removal facilitates delineation of the hillock topography, promoting development of the same symmetric rosette configurations as in greater (micrometer-sized) indentations.

5. Plastic bulging of the material against the indenter tip produces well-developed nanoimprints. The mechanisms for nanoscale bulging are: (i) recurrent slip trace emergence throughout the contact surface, favored by the inception of highly mobile dislocation networks in large stacking-fault energy FCC crystals; (ii) pileup of the material around the imprint vertexes in FCCs; and (iii) surface emergence of nanotwin patterns in BCC nanoimprints -as in the case of (111) surfaces. These mechanisms 
profoundly affect upon the atomic trajectories of the surface atoms, gradually leading to material bulging with tip penetration. Nanoscale bulging is characterized by a gradual increase in parameter $\overline{c^{2}}$ from the perfectly elastic lower limit of 0.5 prior to defect inception. While the value of $\overline{c^{2}}$ at presently imposed maximum penetration is usually greater in FCCs than in BCCs, attainment of the aforementioned twin structure in (111) BCC surfaces enhances bulging to a point where $\overline{c^{2}}$ becomes similar to that produced by recurrent slip in FCC Al. Our simulations in $\mathrm{Cu}$ nanocontacts further suggest that bulging saturation entails at different values of $\overline{c^{2}}$ depending on the uniaxial strain hardening response of the crystal. Finally, parameter $c_{\max }^{2}$ is used in this work to account for the distinctive massive elasto-plastic rebound induced at the nanocontact boundary $(r \gtrsim a)$ upon indenter tip removal.

Acknowledgements: Financial support was provided by Ministerio de Economía y Competitividad through Grant MAT2015-71347-P to JA; CENTEM projects CZ.1.05/2.1.00/03.0088 and PLUS LO1402 to J.O; and project SGS-2016-059 to J.V.

\section{References}

[1] W. Zielinski, H. Huang and W. Gerberich, Microscopy and microindentation mechanics of single crystal Fe-3 wt.\%Si: Part II. TEM of the indentation plastic zone, J. Mater. Res. 8 (1993) 1300-1310.

[2] K. Nibur and D. Bahr, Identifying slip systems around indentations in FCC metals, Scripta. Mater. 49 (2003) 1055-1060.

[3] P. Peralta, R. Ledoux, R. Dickerson, Characterization of surface deformation around Vickers indents in monocrystalline materials,M. Hakik and P. Dickerson, Metall. Mater. Trans. A 35A (2004) 2247-2255.

[4] Y. Wang, D. Raabe, C. Klüber and F. Roters, Orientation dependence of nanoindentation pile-up patterns and of nanoindentation microtextures in copper single crystals Acta Mater. 52 (2004) 2229-2238.

[5] K. Nibur, D. Bahr and B. Somerday, Hydrogen effects on dislocation activity in austenitic stainless steel, Acta Mater. 54 (2006) 2677-2684.

[6] M. Biener, J. Biener, A. Hodge and A. Hamza, Dislocation nucleation in bcc Ta single crystals studied by nanoindentation, Phys. Rev. B 76 (2007) 165422. 
[7] B. Eidel, Crystal plasticity finite-element analysis versus experimental results of pyramidal indentation into (001) fcc single crystal, Acta Mater. 59 (2011) 1761-1771.

[8] Y. Xia, H. Bei, Y. Gao, D. Catoor and E. George, Investigation of deformation and failure mechanisms in nanoindentation mechanics, Mater Sci Eng. A 611 (2014) 177-187.

[9] E. Renner, Y. Gaillard, F. Richard, F. Amiot and P. Delobelle, Sensitivity of the residual topography to single crystal plasticity parameters in Berkovich nanoindentation on FCC nickel, Int. J. Plasticity 77 (2016) 118-140.

[10] M.M. Chaudhri, Chapter 70 Dislocations and Indentations, in: F.R.N. Nabarro and J. P. Hirth (Eds.) Dislocations in Solids, Vol. 12, Elsevier, 2004, pp. 447-550.

[11] Y. Gaillard, C. Tromas and J. Woirgard, Study of the dislocation structure involved in a nanoindentation test by atomic force microscopy and controlled chemical etching, Acta Mater. 51 (2003) 1059-1065.

[12] D. Grabco, B. Pushcash, M. Dyntu and O. Shikimaka, Thermal evolution of deformation zones around microindentations in different types of crystal, Phil. Mag. A 82 (2002) 2207-2215.

[13] S. Lloyd, A. Castellero, F. Giuliani, Y. Long, K. Mclaughlin, J. Molina-Aldareguia, N. Stelmashenko, L. Vandeperre and W. Clegg, Observations of nanoindents via crosssectional transmission electron microscopy: A survey of deformation mechanisms, P. Roy. Soc. A-Math. Phy. 461 (2005) 2521-2543.

[14] J. Jin, S. Shevlin and Z. Guo, Multiscale simulation of onset plasticity during nanoindentation of Al (001) surface, Acta Mater. 56 (2008) 4358-4368.

[15] N. P. Daphalapurkar and K. Ramesh, Orientation dependence of the nucleation and growth of partial dislocations and possible twinning mechanisms in aluminum, J. Mech. Phys. Solids 60 (2012) 277-294.

[16] C. Weinberger and W. Cai, Surface-controlled dislocation multiplication in metal micropillars, P. Natl. Acad. Sci. USA 105 (2008) 14304-14307.

[17] J. Alcalá, R. Dalmau, O. Franke, M. Biener, J. Biener and A. Hodge, Planar Defect Nucleation and Annihilation Mechanisms in Nanocontact Plasticity of Metal Surfaces, Phys. Rev. Lett. 109 (2012) 075502.

[18] R. Hill, B. Störakers and A. Zdunek, A theorical study of the Brinell hardness test, Proc. Royal Soc. London Ser-A 423 (1989) 301-330.

[19] J. Alcalá, A. Barone and M. Anglada, Influence of plastic hardening on surface deformation modes around Vickers and spherical indents, Acta Mater. 48 (2000) 34513464 .

[20] J. Alcalá and D. E. de los Ojos, Reassessing spherical indentation: Contact regimes and mechanical property extractions, Int. J. Solids. Struct. 47 (2010) 2714-2732.

[21] Y. Mishin, D. Farkas, M. Mehl and D. Papaconstantopoulos, Interatomic potentials for monoatomic metals from experimental data and ab initio calculations, Phys. Rev. B 59 (1999) 3393-3407.

[22] Y. Li, D. Siegel, J. Adams and X.-Y. Liu, Embedded-atom-method tantalum potential developed by the force-matching method, Phys. Rev. B 67 (2003) 1251011.

[23] M. I. Mendelev, S. Han, D. J. Srolovitz, G. J. Ackland, D. Y. Sun and M. Asta, Development of new interatomic potentials appropriate for crystalline and liquid iron, 
Phil. Mag. 83 (2003) 3977-3994.

[24] L. Smith, J. A. Zimmerman, L. M. Hale and D. Farkas, Molecular dynamics study of deformation and fracture in a tantalum nano-crystalline thin film, Model. Simul. Mater. Sci. Eng. 22 (2014) 045010.

[25] D. Ward, D. Farkas, J. Lian, W. Curtin, J. Wang, K.-S. Kim and Y. Qi, Engineering size-scaling of plastic deformation in nanoscale asperities, P. Natl. Acad. Sci. USA 106 (2009) 9580-9585.

[26] Y. Gao, C. J. Ruestes, D. R. Tramontina and H. M. Urbassek, Comparative simulation study of the structure of the plastic zone produced by nanoindentation, J. Mech. Phys. Solids 75 (2015) 58-75.

[27] C. Ruestes, A. Stukowski, Y. Tang, D. Tramontina, P. Erhart, B. Remington, H. Urbassek, M. Meyers and E. Bringa, Atomistic simulation of tantalum nanoindentation: Effects of indenter diameter, penetration velocity, and interatomic potentials on defect mechanisms and evolution, Mat. Sci. Eng. A 613 (2014) 390-403.

[28] C. Begau, A. Hartmaier, E. George and G. Pharr, Atomistic processes of dislocation generation and plastic deformation during nanoindentation, Acta Mater. 59 (2011) 934942.

[29] R. Kositski and D. Mordehai, Depinning-controlled plastic deformation during nanoindentation of BCC iron thin films and nanoparticles, Acta Mater 90 (2015) 370-379.

[30] T. Remington, C. Ruestes, E. Bringa, B. Remington, C. Lu, B. Kad and M. Meyers, Plastic deformation in nanoindentation of tantalum: A new mechanism for prismatic loop formation, Acta Mater. 78, (2014) 378-393.

[31] I. Salehinia, S. Lawrence and D. Bahr, The effect of crystal orientation on the stochastic behavior of dislocation nucleation and multiplication during nanoindentation, Acta Mater. 61 (2013) 1421-1431.

[32] C. Kelchner, S. Plimpton and J. Hamilton, Dislocation nucleation and defect structure during surface indentation, Phys. Rev. B 58 (1998) 11085-11088.

[33] G. Ziegenhain, A. Hartmaier and H. Urbassek, Pair vs many-body potentials: Influence on elastic and plastic behavior in nanoindentation of fcc metals, J. Mech. Phys. Solids 57 (2009) 1514-1526.

[34] G. Ziegenhain, H. Urbassek and A. Hartmaier, Influence of crystal anisotropy on elastic deformation and onset of plasticity in nanoindentation: A simulational study, $\mathrm{J}$. Appl. Phys. 107 (2010) 061807.

[35] W. Han, Z. Zhang, S. Wu and S. Li, Combined effects of crystallographic orientation, stacking fault energy and grain size on deformation twinning in fcc crystals, Phil. Mag.

88 (2008) 3011-3029.

[36] D. Bufford, Y. Liu, Y. Zhu, Z. Bi, Q. Jia, H. Wang and X. Zhang, Formation Mechanisms of High-density Growth Twins in Aluminum with High Stacking-Fault Energy, Mat. Res. Lett. 1 (2013) 51-60.

[37] K. Kang, V.V. Bulatov, W. Cai, Singular orientations and faceted motion of dislocations in body-centered cubic crystals, P. Natl. Acad. Sci. USA 109 (2012) 1517415178 .

[38] P.A. Gordon, T. Neeraj, Y. Li, J. Li, Screw dislocation mobility in BCC metals: the 
role of the compact core on double-kink nucleation, Modelling Simul. Mater. Sci. Eng. 18 (2010) 085008.

[39] S. Kucharski and D. Jarzabek, Depth dependence of nanoindentation pile-up patterns in copper single crystals, Met. Trans. A. (2014) 4997-5008.

[40] D. H. Warner, W. A. Curtin and S. Qu, Rate dependence of crack-tip processes predicts twinning trends in f.c.c. metals. Nature Materials 6 (2007) 876 - 881. 

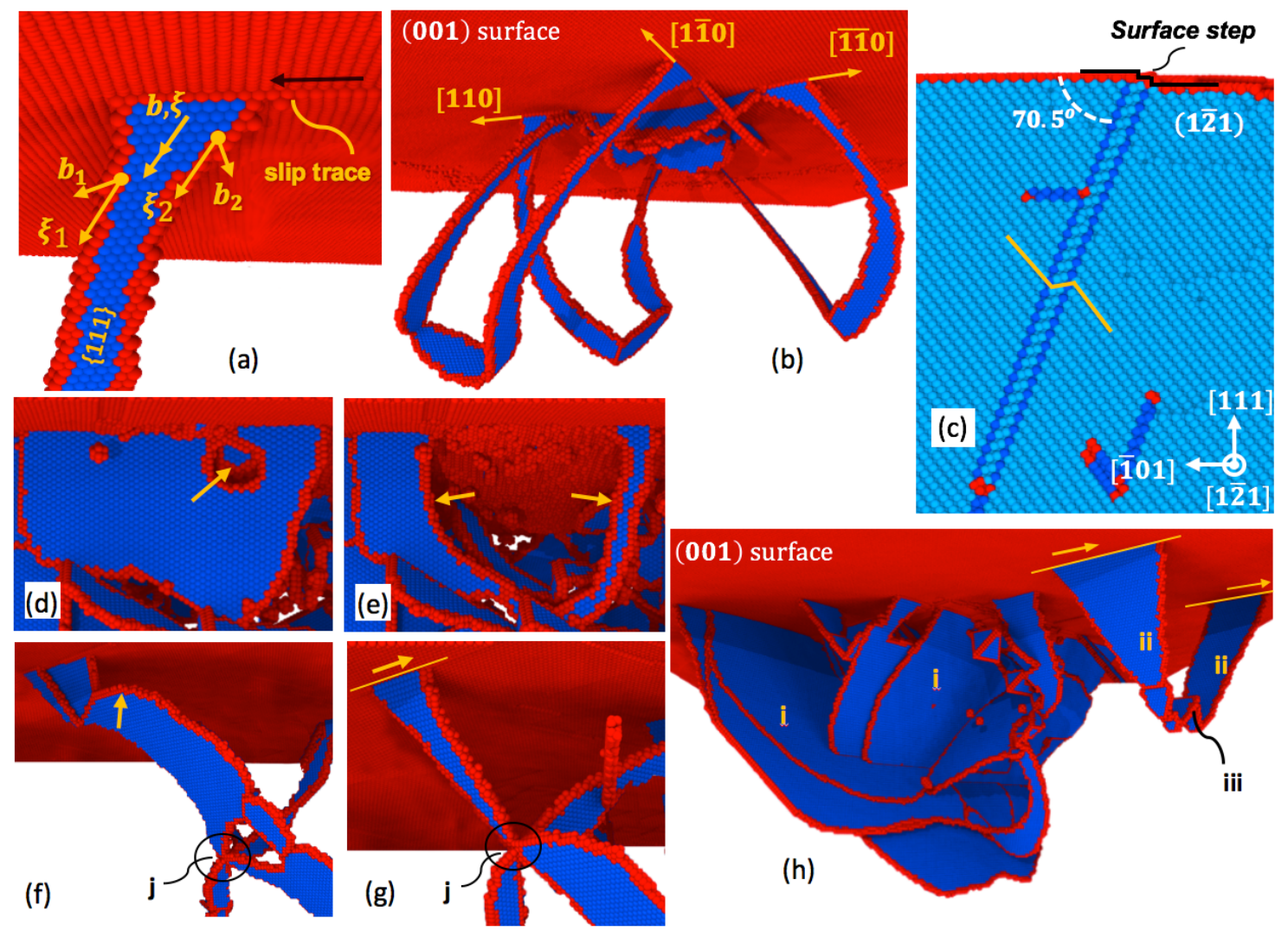

Fig. 1: Onset of slip traces in FCC copper $(T=77 \mathrm{~K})$. (a) Slip trace produced by the passage of a screw dislocation dissociated into leading (1) and trailing (2) segments separated by a stacking-fault (blue) ribbon. (b) Nucleation of prismatic loops producing $<110>$ slip traces. (c) Cross-sectional view of a nanotwin underneath a (111) surface; yellow lines indicate parent and twined crystal orientations. (d) and (e) Nanotwin annihilation (marked in (d)) resulting in the emission of two dislocation segments (marked in (e)). (f) and (g) Surface dislocation emission involving four dislocation arms that revolve around junction " $\mathrm{j}$ ". The process involves gliding of marked dislocation in (f) producing marked trace in (g). (h) Interception of twins labelled "ij" underneath a (001) surface, which results in strong slip trace localization at the surface. Development of dislocation structure labelled "ii" is further shown, where arrows indicate gliding direction, and label "iii" points at collinear interactions.

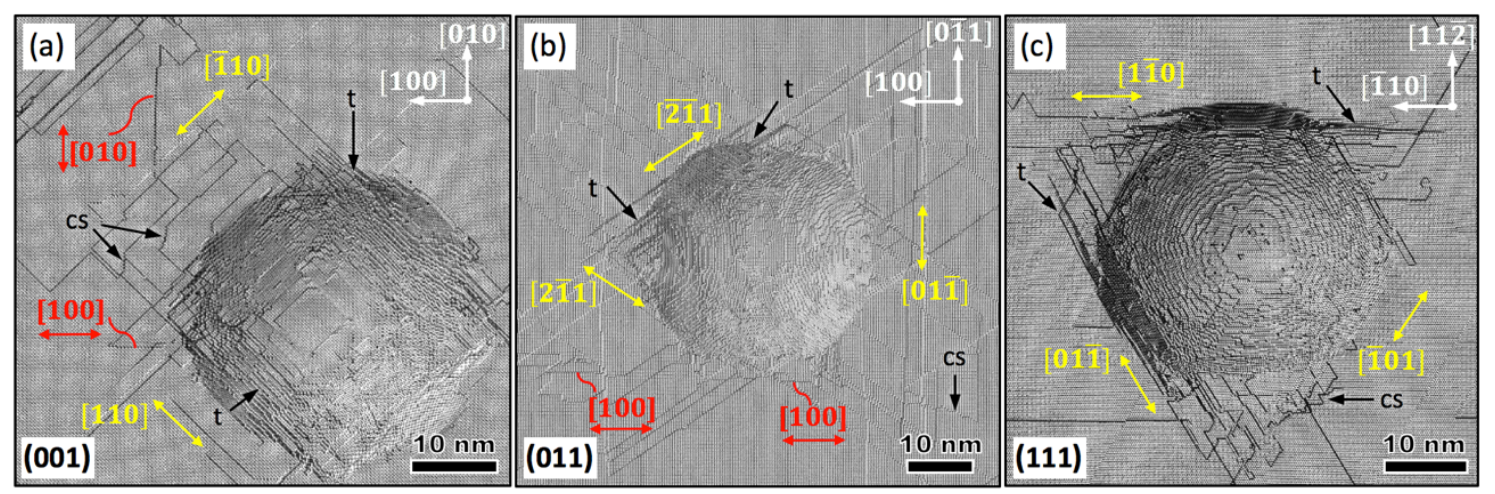

Fig. 2: Slip traces in (001), (011) and (111) FCC Cu surfaces $(T=77 \mathrm{~K} ; D=48 \mathrm{~nm})$. Yellow arrows mark preferential slip directions, where twinning is labelled "t" and "cs" denotes cross-slip. Red arrows indicate apparent non-crystallographic slip traces. 


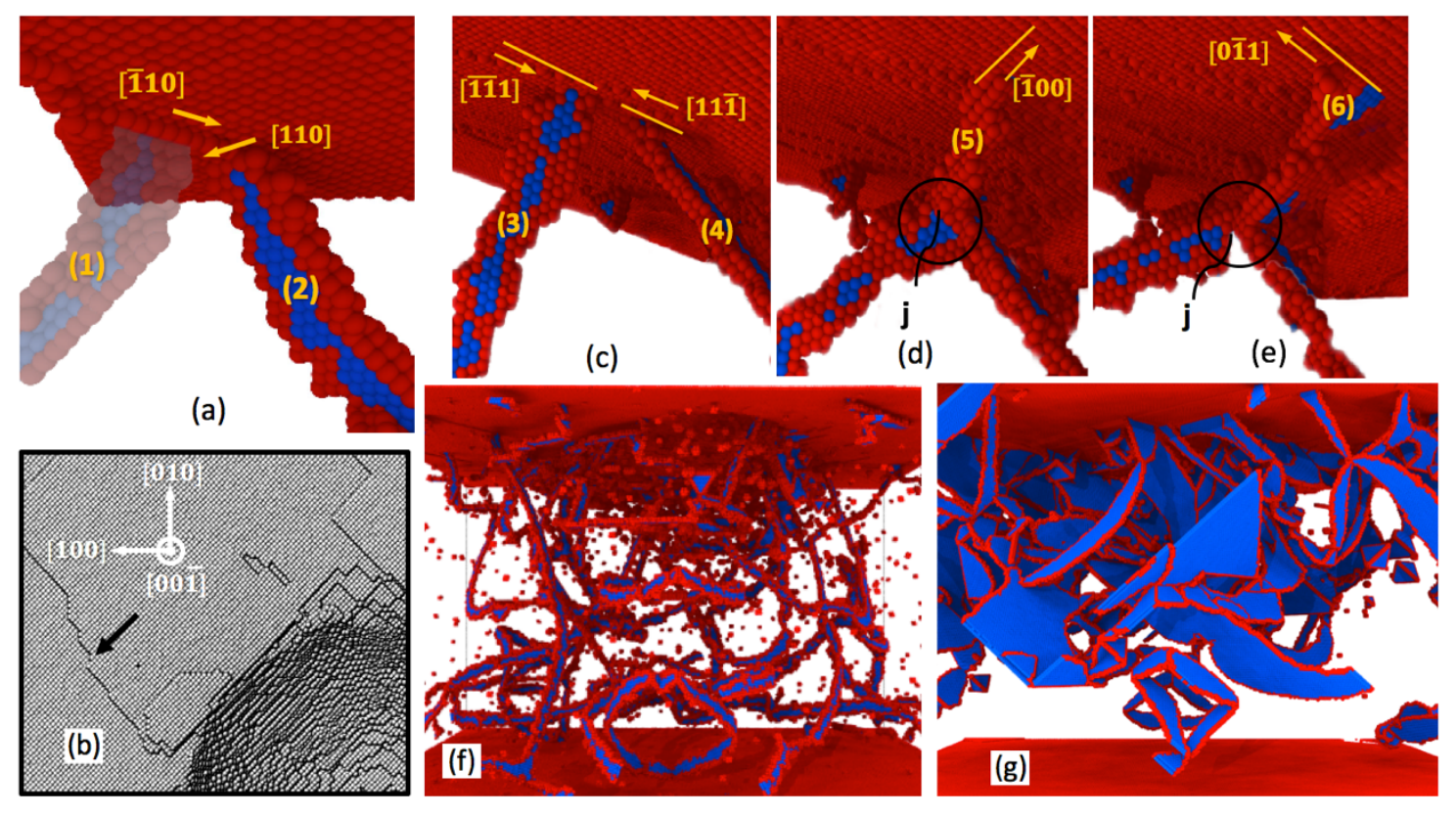

Fig. 3: Cross-slip in FCC Al at 300 K. (a) Pure screw dislocation originally located at "1" cross-slips into location " 2 ". (b) Serrated trace introduced by the cross-slip event in (a). (c) - (e) Development of an apparently non-crystallographic $<001>$ slip trace. Interaction of dislocation segments " 3 " and " 4 " in (c) forms segment " 5 " pinned at junction " $\mathrm{j}$ " in (d). Segment " 5 " is a constricted, full dislocation, that cross-slips producing a trace that is globally oriented along the [100] (non-crystallographic) direction. Dissociation of segment " 5 " into more energetically favorable Shockley partials " 6 " in (e) finally suppresses cross-slip. (f) Linear defect network in Al. (g) Planar-like network in Cu where the large staking fault ribbons (blue) limit cross-slip.

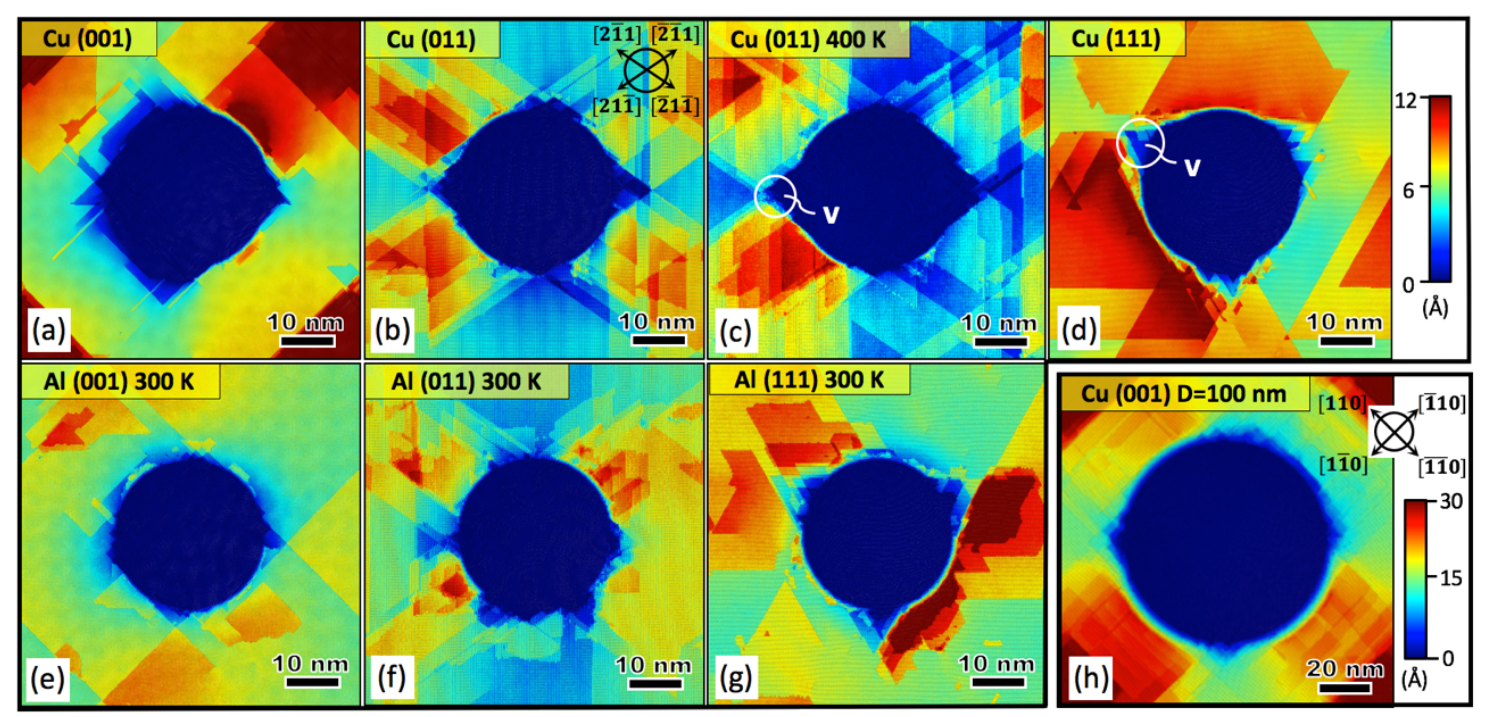

Fig. 4: Pileup topographies in $\mathrm{FCC} \mathrm{Cu} \mathrm{(a)} \mathrm{-} \mathrm{(d)} \mathrm{and} \mathrm{Al}(\mathrm{e})$ - (g). Scale bar refers to surface elevations (red) and depressions (blue). Formation of four-fold rosette symmetries is shown for all (011) indentations $(D=48 \mathrm{~nm})$ and the (001) indentation with $D=100 \mathrm{~nm}$ in (h). Notice development of imprint vertexes (marked "v") around the nanoimprints. The influence of the more serrated slip in $\mathrm{Al}$ as compared to in $\mathrm{Cu}$ is further illustrated by comparison between (b) and (f). Temperature is set at $77 \mathrm{~K}$ for all $\mathrm{Cu}$ surfaces except part (c) that is for $400 \mathrm{~K}$. The $\mathrm{Al}$ surfaces are indented at $300 \mathrm{~K}$. 


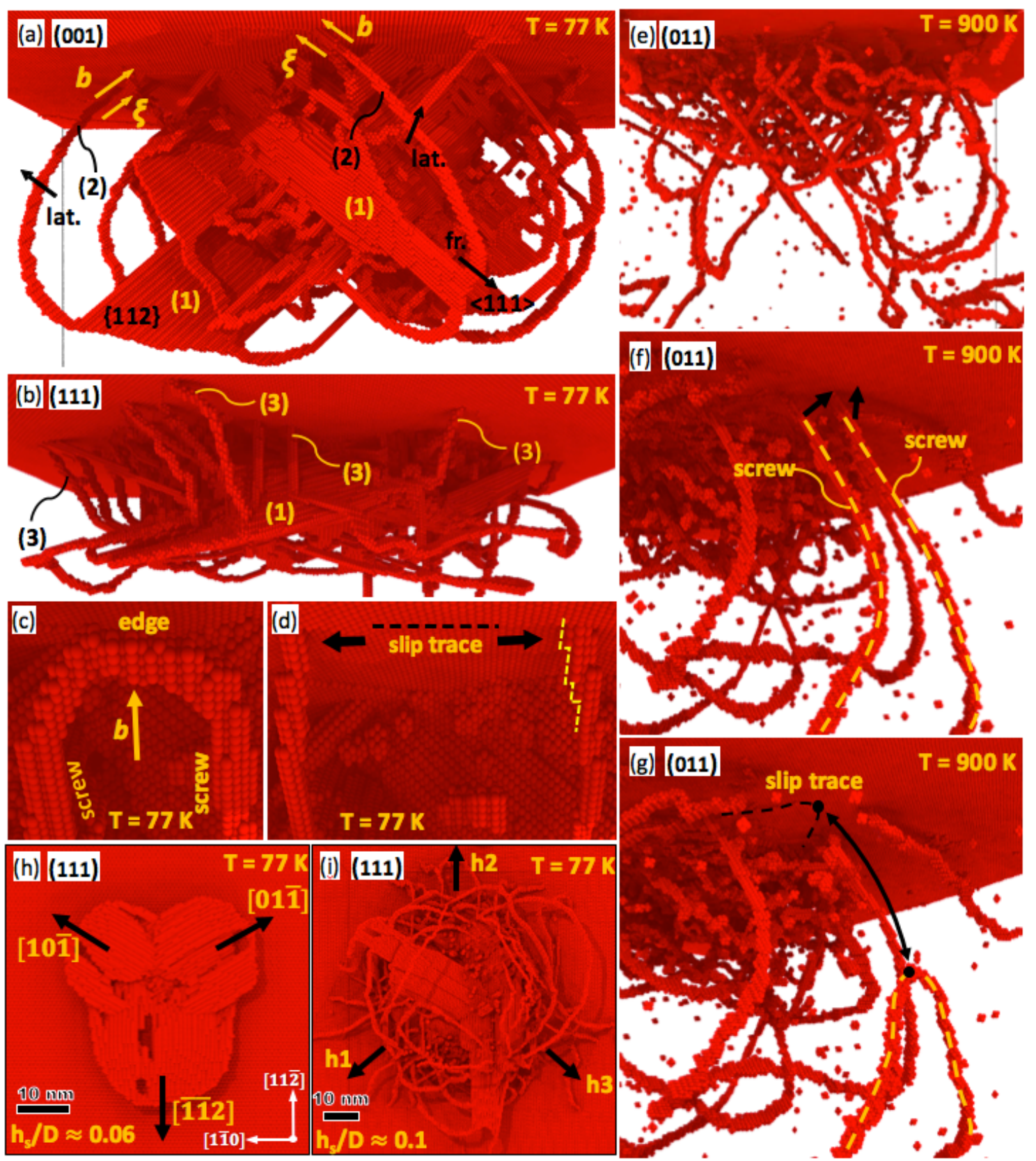

Fig. 5: Slip trace formation mechanisms in BCC Ta $(D=48 \mathrm{~nm})$. (a) Defect network where partial annihilation of $\{112\}$ twins "(1)" leads to the lateral (lat.) emission of screw dislocations "(2)" intercepting the surface, as well as the frontal (fr.) emission of edge segments towards the subsurface. (b) Heterogeneous dislocation loop nucleation where precursory twins labeled "(1)" produce screw segments labeled "(3)". (c) and (d) Surface interception of a nucleated loop, where the edge segment produces a straight trace that is extended by the partnering screws through dislocation kinking -marked by dashed yellow line. (e) Linear defect network incepted at elevated temperatures. (f) Expansion of a surface truncated dislocation loop in the direction of the arrows produces dislocation segment annihilation. Segment annihilation entails along the arrow in (g), introducing a closed slip trace at the surface and dislocation loop in the subsurface. (h) Bottom view of the three-fold twin structure that produces marked bulging in (111) surfaces. (i) Gliding of screw dislocations emitted from the twin structure in (h) introduces hillocks h1, h2 and h3 (further shown in Figs. 6(f) and 6(g)). 


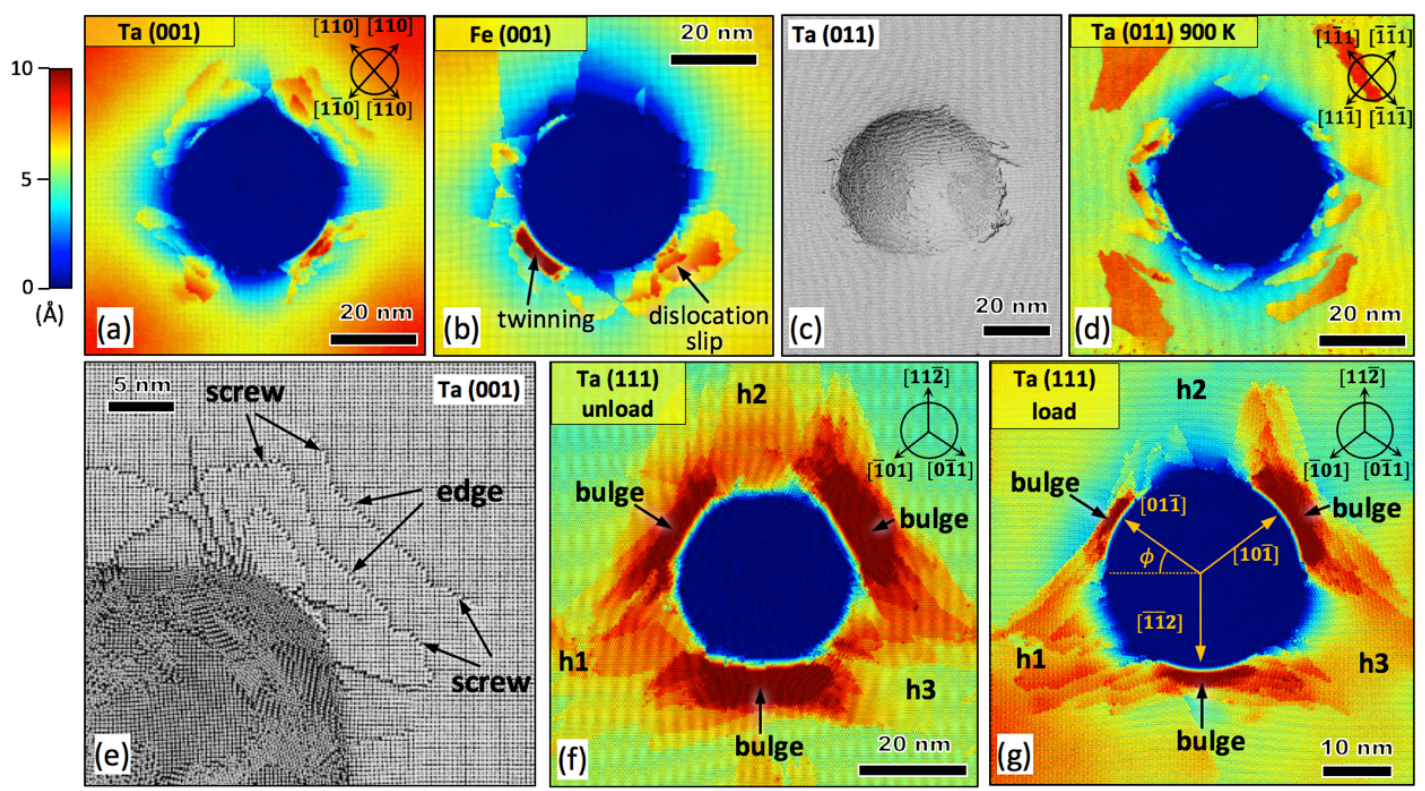

Fig. 6: Pileup topographies in BCC nanocontacts at $77 \mathrm{~K}(D=48 \mathrm{~nm})$. Scale bar denotes surface elevations (red) and depressions (blue). (a) Formation of confined hillocks in (001) nanoimprints through interplay between screw and edge dislocation gliding marked in (e). (b) Material pileup due to twinning and dislocation slip. (c) and (d) Influence of increasing temperature upon the outward spreading of dislocation loops, contributing to terrace formation. (f) and (g) Development of a three-fold symmetric rosette arrangement comprised by hillocks $h 1, h 2$ and $h 3$ in loaded and unloaded states at $\approx 60^{\circ}$ from the regions of maximum bulging (pileup). The bulged regions are induced by the three-fold twin structure in Fig. 5(h). Hillock development is due to gliding of surfaced screw dislocations sideward from the bulged regions (also illustrated in Fig. 5(i)).
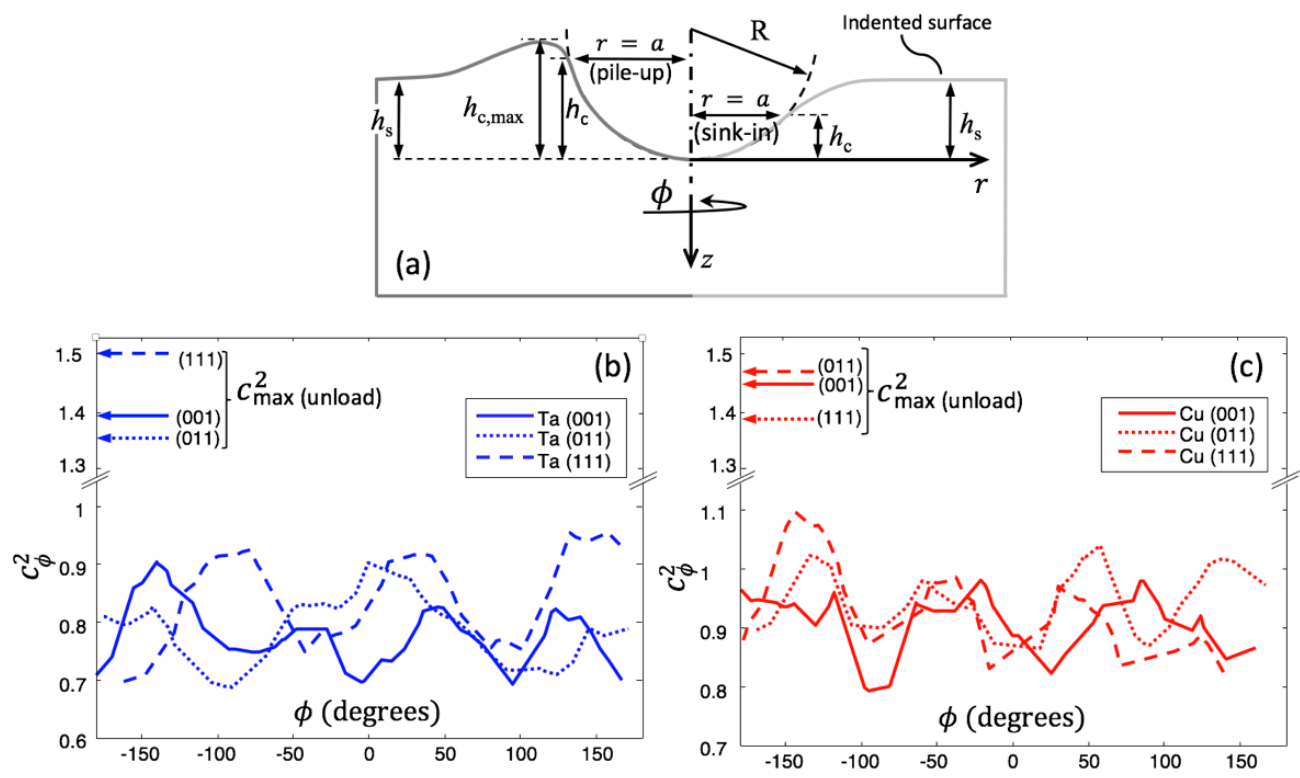

Fig. 7: Plastic bulging around the indenter-tip $(D=48 \mathrm{~nm} ; T=77 \mathrm{~K})$. (a) Associated nomenclature under material pileup and sinking-in effects. (b) and (c) Variation of pileup parameter $c_{\phi}^{2}$ around the imprint for $\mathrm{Ta}$ and $\mathrm{Cu}$ surfaces, respectively, at maximum imposed tip penetration. Angle $\phi$ increases clockwise for all orientations following Fig. $6(\mathrm{~g})$. Parameter $c_{\max }^{2}$ upon tip unloading is also shown. See text for details. 

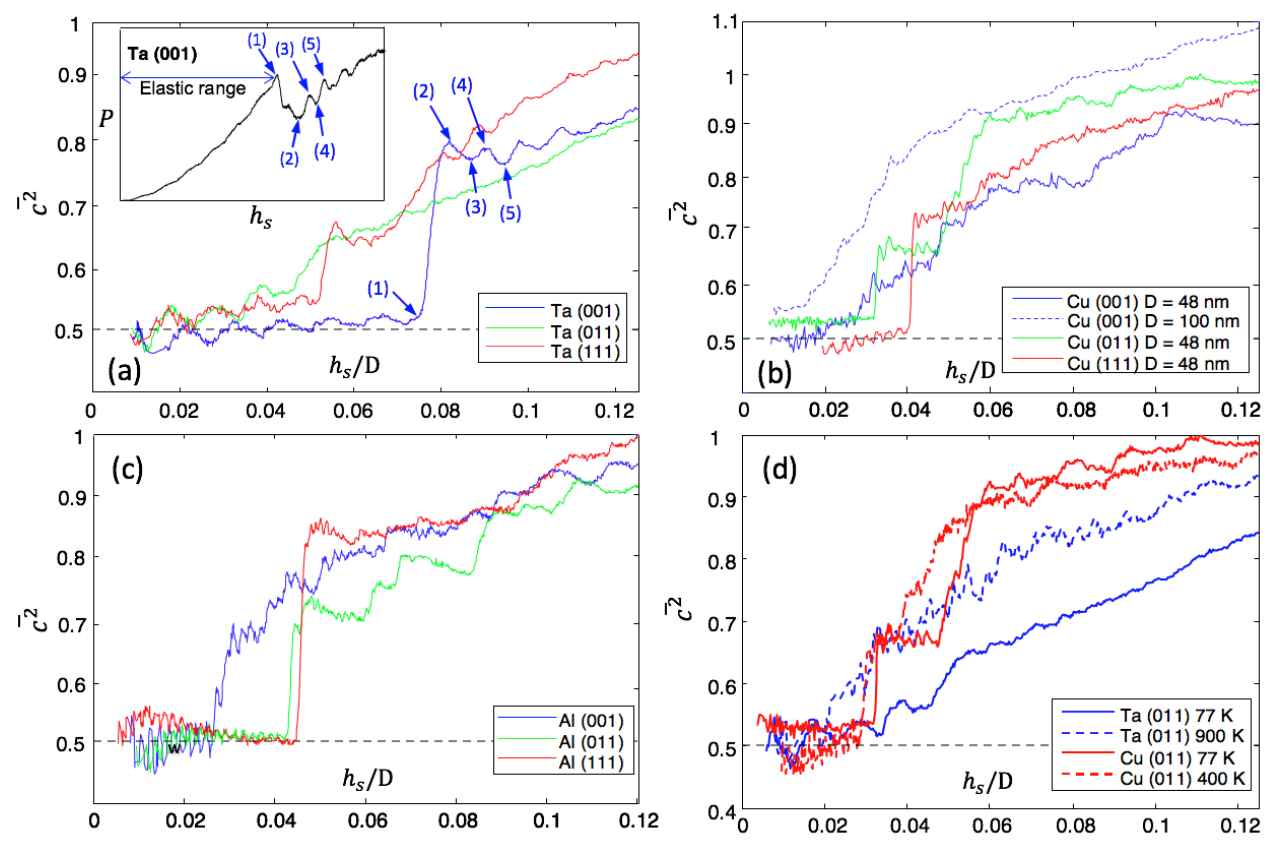

Fig. 8: Evolution of $\overline{c^{2}}$ with increasing normalized penetration $h_{s} / D$ for Ta (a), $\mathrm{Cu}$ (b) and $\mathrm{Al}$ (c) surfaces $(D=48 \mathrm{~nm} ; T=77 \mathrm{~K})$. Inset to part (a) shows the associated applied load-penetration depth $\left(P-h_{s}\right)$ curve, where the load drops marking plastic bursts lead to abrupt raises in $\overline{c^{2}}$. Part (b) includes results for $D=100 \mathrm{~nm}$. Part (d) illustrates influence of temperature.
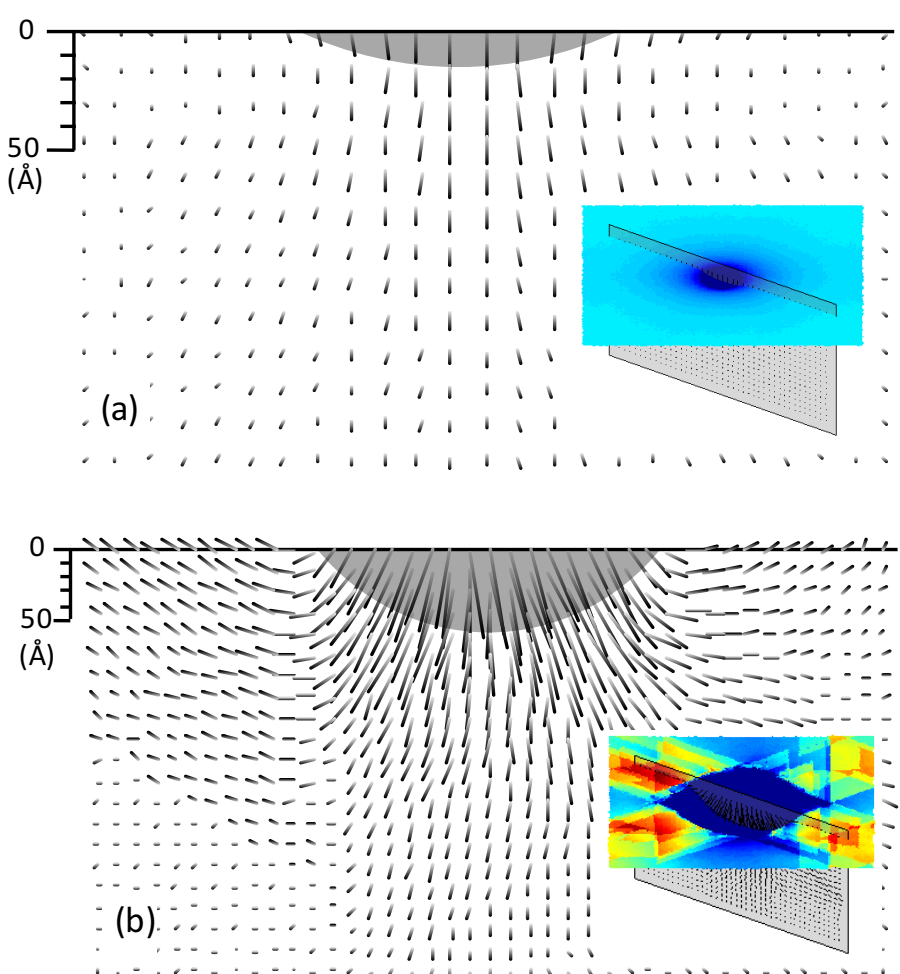

Fig. 9: Atomic displacement fields for (011) $\mathrm{Cu}$ nanocontacts along the marked crosssectional views in the insets. Arrow length is proportional to atomic displacement. (a) Early elastic response with pronounced sinking-in, $\overline{c^{2}}=0.5$. (b) Uplift of the surface atoms due to the incepted defect network at maximum imposed penetration. 

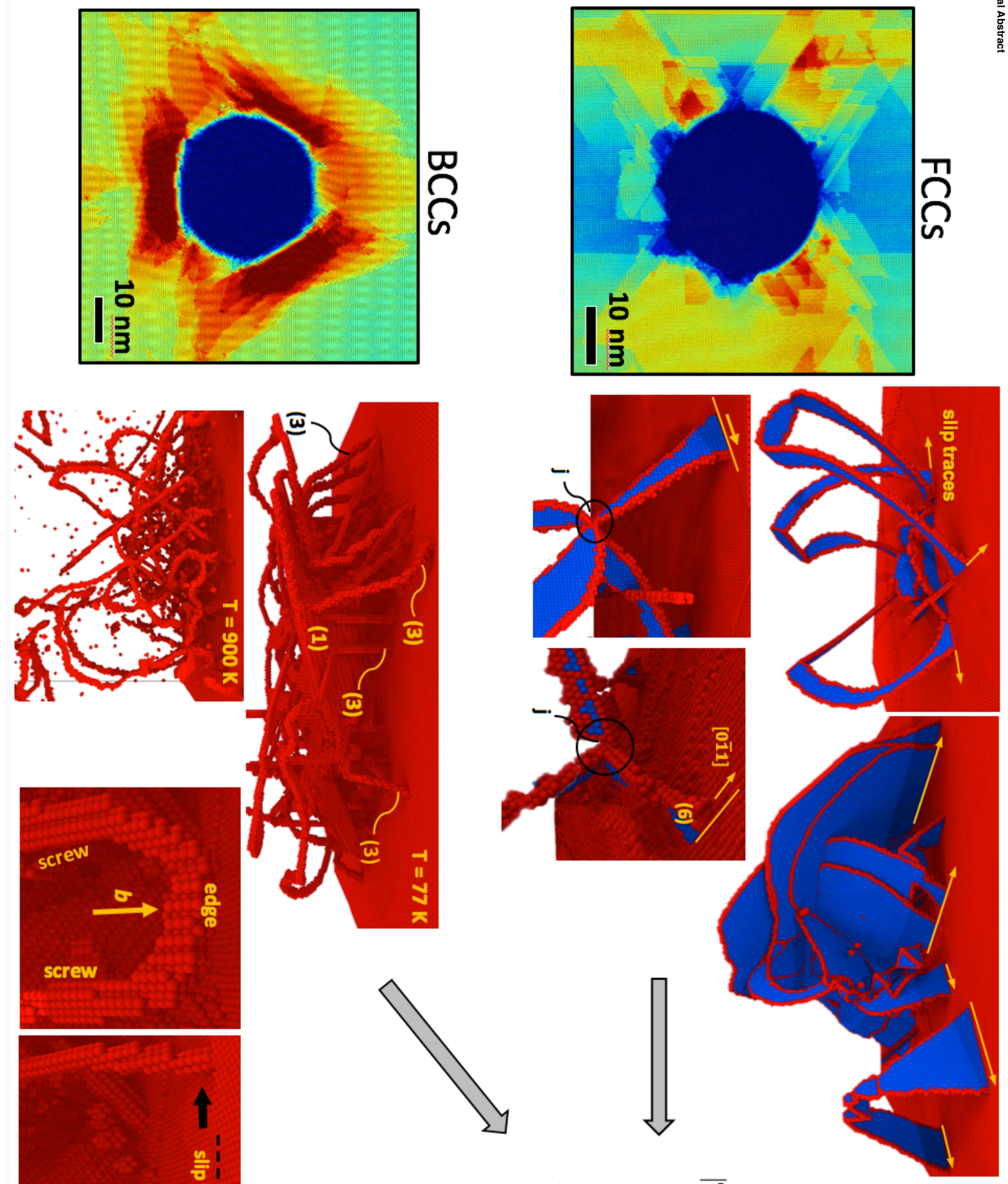

screw
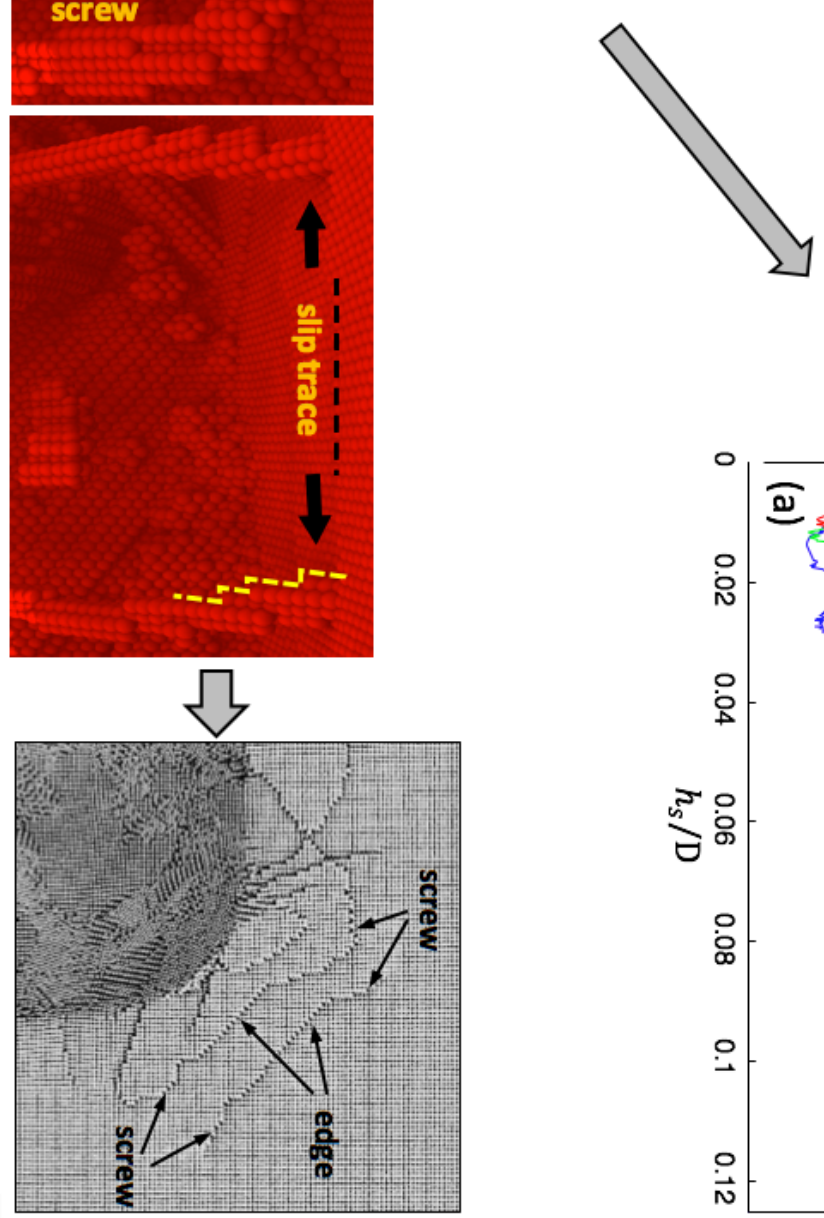

pileup parameter, $\overline{c^{2}}$

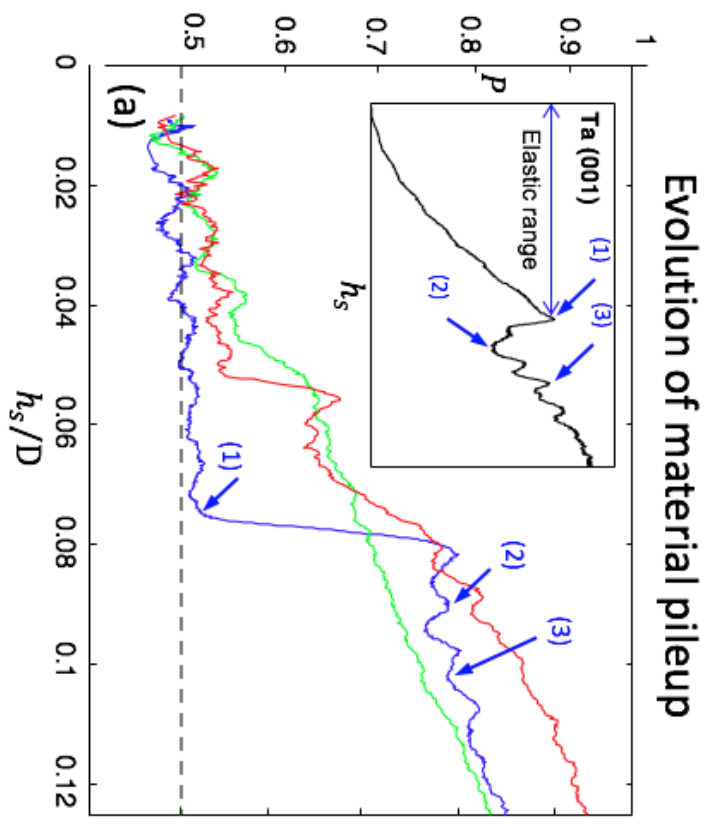

